\title{
Universal formula for the mean first passage time in planar domains
}

\author{
Denis S. Grebenkov ${ }^{1, *}$ \\ ${ }^{1}$ Laboratoire de Physique de la Matière Condensée (UMR 7643), \\ CNRS - Ecole Polytechnique, University Paris-Saclay, 91128 Palaiseau, France
}

(Dated: Received: September 1, 2018/ Revised version:)

\begin{abstract}
We derive a general exact formula for the mean first passage time (MFPT) from a fixed point inside a planar domain to an escape region on its boundary. The underlying mixed Dirichlet-Neumann boundary value problem is conformally mapped onto the unit disk, solved exactly, and mapped back. The resulting formula for the MFPT is valid for an arbitrary space-dependent diffusion coefficient, while the leading logarithmic term is explicit, simple, and remarkably universal. In contrast to earlier works, we show that the natural small parameter of the problem is the harmonic measure of the escape region, not its perimeter. The conventional scaling of the MFPT with the area of the domain is altered when diffusing particles are released near the escape region. These findings change the current view of escape problems and related chemical or biochemical kinetics in complex, multiscale, porous or fractal domains, while the fundamental relation to the harmonic measure opens new ways of computing and interpreting MFPTs.
\end{abstract}

PACS numbers: 02.50.-r, 05.60.-k, 05.10.-a, 02.70.Rr

Keywords: Escape problem, First passage time, Mixed boundary condition, Conformal mapping

How long does it take for diffusing species to exit from an irregular domain or to initiate a reaction on a catalytic site or an enzyme? Since the first contribution by Lord Rayleigh [1], the first-passage phenomena have attracted much attention [2 [5], and have been applied to numerous chemical [6] and biological [7] problems such as diffusion-influenced ligand binding to receptors on cell surfaces [8, 9], receptor trafficking in synaptic membranes [10], diffusion in cellular microdomains [11], or foraging strategies of animals [12], to name but a few. Most analytical results were obtained for the mean first passage time (MFPT) to a small region on the boundary, which can represent a specific target, a catalytic germ, an active site, a channel or an exit to the outer space [5, 1321]. For a "regular" planar domain $\Omega$ (whose perimeter $|\partial \Omega|$ and linear size $|\Omega|^{\frac{1}{2}}$ are comparable, see [5]), the MFPT, averaged over uniformly distributed starting points, was shown to be $\frac{|\Omega|}{\pi D}[\ln (1 / \varepsilon)+O(1)]$, where $D$ is the diffusion coefficient, $|\Omega|$ is the area of the domain, and $\varepsilon=|\Gamma| /|\partial \Omega| \ll 1$ is the perimeter of the escape region $\Gamma$ divided by the perimeter of the boundary $\partial \Omega$ 14]. In the special case of a disk, Singer et al. also showed that the MFPT from a fixed starting point (e.g., the center) exhibits similar $\ln (1 / \varepsilon)$ behavior [15]. Since these seminal works, the logarithmic divergence of the MFPT with respect to the normalized perimeter $\varepsilon$ has become a common paradigm (see the review [5] and references therein).

In this letter, we show that this paradigm is incomplete for general domains and can be strongly misleading when the starting point is fixed. We derive the exact formula for the MFPT from a fixed point $x_{0}$ to a connected escape region $\Gamma$ on the boundary of any simply connected (i.e., without "holes") planar domain $\Omega$. This formula

*Electronic address: denis.grebenkov@polytechnique.edu is not restricted to the narrow escape limit $\varepsilon \ll 1$ and is valid for an arbitrary space-dependent diffusion coefficient. Most importantly, we reveal an earlier unnoticed fundamental relation between the MFPT and the harmonic measure of the escape region, $\omega=\omega_{x_{0}}(\Gamma)$, i.e., the probability of arriving at the escape region $\Gamma$ before hitting the remaining part of the boundary [22].

Before proceeding to rigorous results, we start with two examples of "nonregular" domains casting doubts on the normalized perimeter $\varepsilon$ as the universal small parameter. If the domain is a thin long rectangle $[0, L] \times[0, h]$, the MFPT to the left short edge from a starting point $x_{0}=\left(x_{0}^{1}, x_{0}^{2}\right)$ is equal to $\left(L x_{0}^{1}-\frac{1}{2}\left[x_{0}^{1}\right]^{2}\right) / D$. Being independent of $h$, this MFPT is thus not determined by the normalized perimeter $\varepsilon=\frac{h}{2(L+h)}$, even if the latter is very small. In the second example, one takes a disk and replaces a small arc of its boundary by a very corrugated (e.g., fractal) curve. Keeping the diameter $\delta$ of the modified escape region small, one can make its perimeter arbitrarily large. Since the remaining part of the circle is fixed, the ratio $\varepsilon=|\Gamma| /|\partial \Omega|$ can be made close to 1 . When $\delta$ is small, the MFPT should be large, in spite the fact of $\varepsilon \approx 1$. These two very basic examples illustrate the failure of the normalized perimeter of the escape region as a determinant of the MFPT when the starting point is fixed. We will show that the natural characteristic that substitutes the normalized perimeter $\varepsilon$ is the harmonic measure $\omega_{x_{0}}(\Gamma)$.

For Brownian motion starting from an interior point $x_{0}$ of a simply connected planar domain $\Omega$, the MFPT $\mathcal{T}\left(x_{0}\right)$ to a connected escape region $\Gamma$ on the boundary $\partial \Omega$ satisfies the backward Fokker-Planck equation [23]

$$
\Delta \mathcal{T}\left(x_{0}\right)=-\frac{1}{D\left(x_{0}\right)} \quad x_{0} \in \Omega
$$

with mixed Dirichlet-Neumann boundary conditions

$$
\mathcal{T}\left(x_{0}\right)=0 \quad x_{0} \in \Gamma, \quad \partial_{n} \mathcal{T}\left(x_{0}\right)=0 \quad x_{0} \in \partial \Omega \backslash \Gamma,
$$


where $\partial_{n}$ is the normal derivative, $\Delta$ is the Laplace operator, and $D\left(x_{0}\right)$ is the space-dependent diffusion coefficient. According to the Riemann mapping theorem, the unit disk $\mathcal{D}$ can be mapped onto $\Omega$ by a conformal mapping $\phi_{x_{0}}(z): \mathcal{D} \rightarrow \Omega$. We fix two parameters of the conformal map by imposing that the origin of $\mathcal{D}$ is mapped onto the starting point $x_{0}: \phi_{x_{0}}(0)=x_{0}$. Since the conformal mapping preserves the harmonic measure, the preimage of $\Gamma$ is an arc $\gamma$ of the unit circle of length $2 \pi \omega$. Note that the harmonic measure is fully determined by the conformal map. The third parameter of the conformal mapping is fixed by rotating the arc $\gamma$ to be $(-\pi \omega, \pi \omega)$. Setting $\tau(z)=\mathcal{T}\left(\phi_{x_{0}}(z)\right)$ for $z \in \mathcal{D}$, Eqs. (11) 2) are transformed into

$$
\left\{\begin{aligned}
\Delta \tau(z) & =-\left|\phi_{x_{0}}^{\prime}(z)\right|^{2} / D\left(\phi_{x_{0}}(z)\right) & & z \in \mathcal{D}, \\
\tau(z) & =0 & & z \in \gamma, \\
\partial_{n} \tau(z) & =0 & & z \in \partial \mathcal{D} \backslash \gamma .
\end{aligned}\right.
$$

The solution of this mixed boundary value problem can be reduced to dual trigonometric equations whose solutions are well documented 24]. Skipping mathematical details (see SM1), we obtain for any interior starting point $x_{0} \in \Omega$

$$
\mathcal{T}\left(x_{0}\right)=\int_{\mathcal{D}} \frac{d z\left|\phi_{x_{0}}^{\prime}(z)\right|^{2}}{D\left(\phi_{x_{0}}(z)\right)}\left(-\frac{\ln |z|}{2 \pi}+W_{\omega}(z)\right),
$$

with

$$
W_{\omega}(z)=\frac{1}{\pi} \ln \left(\frac{\left|1-z+\sqrt{\left(1-z e^{i \pi \omega}\right)\left(1-z e^{-i \pi \omega}\right)}\right|}{2 \sin (\pi \omega / 2)}\right),
$$

in which the most challenging "ingredient" of the problem, the mixed boundary condition, is fully incorporated through the explicit function $W_{\omega}(z)$. The function $W_{\omega}(z)$ is universal; its dependence on $\Omega, \Gamma$ and $x_{0}$ enters uniquely through the harmonic measure $\omega=\omega_{x_{0}}(\Gamma)$. To return to the domain $\Omega$, the integration variable $z$ is changed to $x=\phi_{x_{0}}(z)$, which yields

$$
\mathcal{T}\left(x_{0}\right)=\int_{\Omega} \frac{d x}{D(x)} \underbrace{\left(-\frac{\ln \left|\phi_{x_{0}}^{-1}(x)\right|}{2 \pi}+W_{\omega}\left(\phi_{x_{0}}^{-1}(x)\right)\right)}_{\text {Green's function }} .
$$

The exact solution (6) is our main result. The two terms are, respectively, (i) the MFPT from $x_{0}$ to the whole boundary $\partial \Omega$, with $\frac{1}{2 \pi} \ln \left|\phi_{x_{0}}^{-1}(x)\right|$ being the Dirichlet Green's function in $\Omega$, and (ii) the contribution from eventual reflections on the remaining part of the boundary, $\partial \Omega \backslash \Gamma$, until reaching the escape region $\Gamma$. The integral form of the solution $\mathcal{T}\left(x_{0}\right)$, which is valid for an arbitrary function $1 / D(x)$, allows one to interpret the expression in parentheses in Eq. (66) as the Green's function of the Laplace operator $-\Delta$ subject to mixed Dirichlet-Neumann boundary condition (2). Numerical implementation of the exact solution (6), its accuracy, and a comparison to conventional numerical methods for computing MFPTs are discussed in SM2. While conformal mappings have been intensively used to solve diffusion-reaction problems (e.g., see [5, 25 34] and references therein), this powerful technique is applied to the mixed boundary value problem (1, 2) for the first time. Prior to this work, no exact solution of this MFPT problem was available, except for a few simple domains 15, 20, 21]. While the proposed approach is limited to planar Brownian diffusion (see the further discussion in SM1), the universality of the exact solution (6) results from the existence of a conformal map for any simply connected planar domain, even with a very irregular (e.g., fractal) boundary. The only mathematical restriction on the domain is that the original problem (11, 2) should be well defined. In the case of the unit disk with a constant diffusivity, our general formula (6) reduces to the earlier result [20]

$$
\mathcal{T}\left(x_{0}\right)=\frac{1-\left|x_{0}\right|^{2}}{4 D}+\frac{\pi}{D} W_{\varepsilon}\left(x_{0}\right)
$$

(see SM3 for the derivation).

The general solution (6) allows one to investigate, for the first time, the impact of heterogeneous diffusivity in MFPT problems defined in nontrivial domains. In spite of its evident importance for biological systems in which the spatial heterogeneity is ubiquitous, only a few analytical results about one-dimensional motion with spacedependent diffusivity are available [35]. Even for simple domains such as disks or rectangles, the spatial dependence of the diffusion coefficient can prohibit the separation of variables or, in the most favorable cases, lead to complicated differential equations whose solutions cannot be expressed in terms of usual special functions. In this light, it is remarkable that the formula (6) provides an exact solution even for the space-dependent diffusivity $D(x)$. One can investigate, e.g., how the lower diffusivity in the actin cortex near the plasma membrane would affect the overall MFPT in flat living cells adhered to a surface, as well as the effect of corrals on membrane diffusion.

When the harmonic measure of the escape region is small, one can derive the perturbative expansion of the MFPT (see SM4):

$$
\begin{aligned}
\mathcal{T}\left(x_{0}\right) & =\frac{|\Omega|}{\pi D_{h}} \ln (1 / \omega) \\
& +\left(\frac{|\Omega| \ln (2 / \pi)}{\pi D_{h}}+\frac{1}{2 \pi} \int_{\Omega} \frac{d x}{D(x)} \ln \frac{\left|1-\phi_{x_{0}}^{-1}(x)\right|^{2}}{\left|\phi_{x_{0}}^{-1}(x)\right|}\right) \\
& +\omega^{2}\left(\frac{\pi|\Omega|}{24 D_{h}}-\int_{\Omega} d x \frac{V_{2}\left(\phi_{x_{0}}^{-1}(x)\right)}{D(x)}\right)+O\left(\omega^{4}\right),
\end{aligned}
$$

with

$$
V_{2}(z)=-\frac{\pi}{8}\left(\frac{z}{(1-z)^{2}}+\frac{\bar{z}}{(1-\bar{z})^{2}}\right) .
$$


The first logarithmic term appears as the leading contribution that involves the harmonic measure of the escape region, $\omega=\omega_{x_{0}}(\Gamma)$, the area of the domain, $|\Omega|$, and the harmonic mean of the space-dependent diffusion coefficient:

$$
\frac{1}{D_{h}}=\frac{1}{|\Omega|} \int_{\Omega} \frac{d x}{D(x)}
$$

(if $D(x)=D$ is constant, then $D_{h}=D$ ). The expansion (8) and its leading term $\frac{|\Omega|}{\pi D_{h}} \ln \left(1 / \omega_{x_{0}}\right)$ present the second main result that allows one to estimate the MFPT directly through the harmonic measure.

In fact, when the starting point $x_{0}$ is not too close to the boundary, the leading term provides a good approximation to the MFPT. To illustrate this point, we generated a planar domain in Fig. Ta by iterative conformal maps (see SM5). In order to emphasize that the normalized perimeter $\varepsilon$ of the escape region must be replaced by the harmonic measure, we fix $\varepsilon=0.035$ and then move the escape region $\Gamma_{s}$ of perimeter $\varepsilon|\partial \Omega|$ along the boundary of the domain, where $s$ is the curvilinear coordinate of the center of $\Gamma_{s}$. Figure $1 \mathrm{~b}$ shows $\mathcal{T}\left(x_{0}\right)$ from Eq. (6) and its leading term $-\frac{|\Omega|}{\pi D} \ln \omega_{x_{0}}\left(\Gamma_{s}\right)$ from Eq. (8), as functions of the location $s$ of the escape region $\Gamma_{s}$ on the boundary for $x_{0}=0$ (we set $D=1$ and use dimensionless units). As expected, the MFPT significantly varies when the escape region moves, showing the dependence on the distance between $x_{0}$ and $\Gamma$ and on the shape of the domain. For instance, the corner at the curvilinear location $s \approx 2.7$ is difficult to access so that the related harmonic measure is very small, while the MFPT exhibits a prominent peak rising up to 14 . In turn, three escape regions at $s \approx 0,3.7,7.3$ are the closest to the starting point and thus easily accessible, resulting in the minima of the MFPT. One can see that the leading term with the harmonic measure provides an excellent approximation to the MFPT. In turn, the conventional leading term $\frac{|\Omega|}{\pi D} \ln (1 / \varepsilon)$ (which is independent of the location) is a poor estimate, in spite of the fact that $\varepsilon$ is small. We conclude that when the starting point is fixed, the harmonic measure of the escape region should substitute the normalized perimeter as the natural small parameter, at least in two dimensions.

When the starting point $x_{0}$ is close to the boundary, the logarithmic term overestimates the MFPT, as illustrated in Fig. 10 for the starting point $x_{0}=0.4590+$ $0.7936 i$. One can see that the logarithmic term accurately captures the behavior of the MFPT when the escape region is close to the starting point $(s$ between 1 and 3 ) while a significant but nearly constant deviation appears for distant escape regions. Here, the contribution from the next-order terms in Eq. (8), in particular, that of order $O(1)$, is comparable to the logarithmic term.

This situation can also be illustrated on the example of a thin rectangle $[0, L] \times[0, h]$ that we discussed at the beginning. In fact, the harmonic measure $\omega$ of the short edge on the left, seen from a point $\left(x_{0}^{1}, h / 2\right)$, is approx-
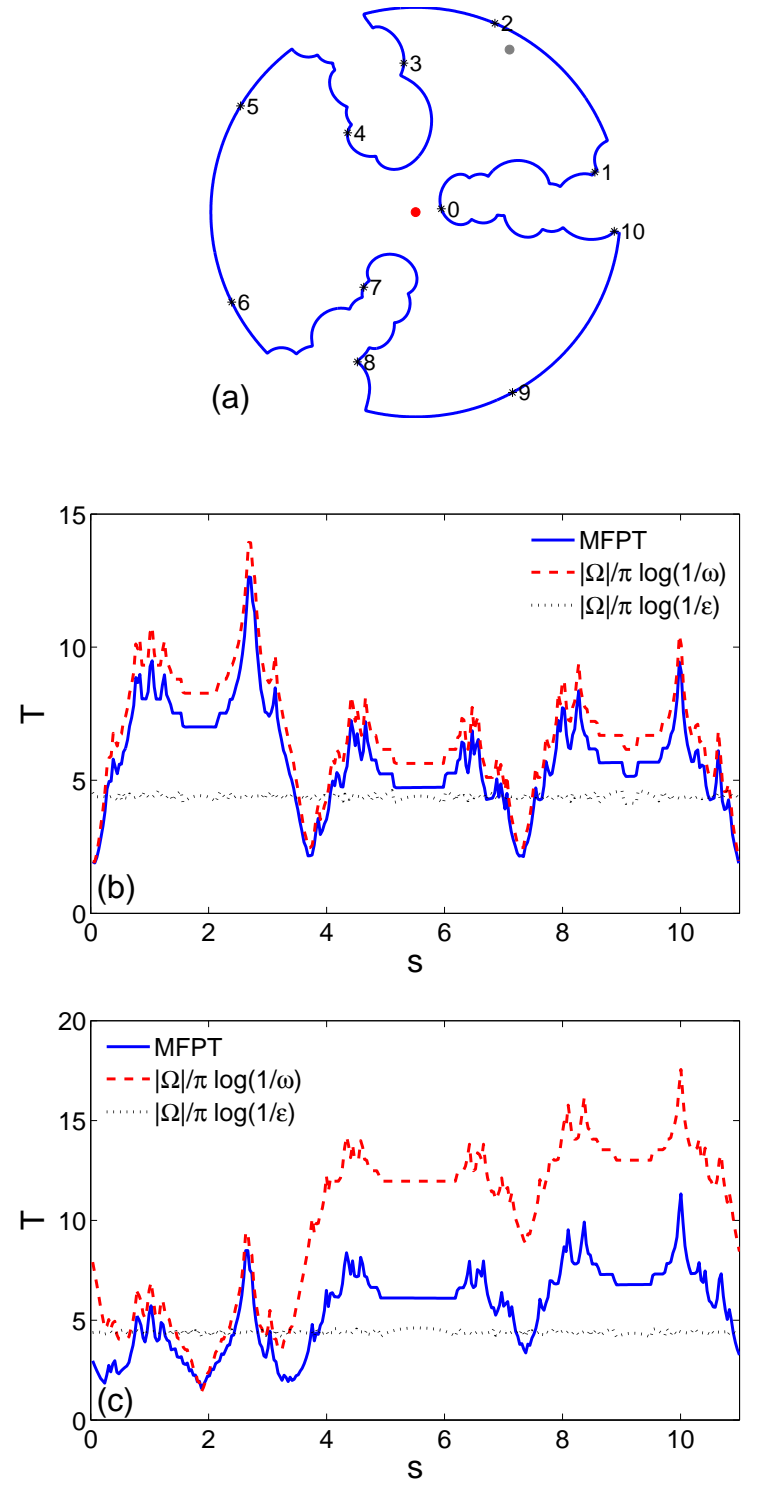

FIG. 1: (Color online) (a) An irregular domain obtained by iterative conformal maps (see SM5). The red and gray circles indicate two considered starting points: $x_{0}=0$ and $x_{0}=0.4590+0.7936 i$; the asterisks with indicated curvilinear coordinates present "milestones" along the boundary; the perimeter, the diameter, and the area of the domain are 10.96, 2 , and 2.39, respectively. (b,c) The MFPT $\mathcal{T}\left(x_{0}\right)$ (solid line), the leading term $\frac{|\Omega|}{\pi D} \ln \left(1 / \omega_{x_{0}}\left(\Gamma_{s}\right)\right)$ (dashed line), and the conventional leading term $\frac{|\Omega|}{\pi D} \ln (1 / \varepsilon)$ (dotted line) as functions of the location $s$ of the escape region $\Gamma_{s}$ on the boundary of the shown domain, with $x_{0}=0$ (b) and $x_{0}=0.4590+0.7936 i$ (c). Note that small fluctuations of the dotted line are related to small variations in the perimeter of the escape region due to discretization. Here, we set $D=1$ and use dimensionless units. 
imately $\exp \left(-\pi x_{0}^{1} / h\right)$ (see SM3). Substitution of this expression into the first term of Eq. (8) yields $L x_{0}^{1} / D$, which is close to the exact MFPT $\left(L x_{0}^{1}-\frac{1}{2}\left[x_{0}^{1}\right]^{2}\right) / D$. This simple example illustrates that (i) even if the harmonic measure $\omega$ is very small, the contribution from the remaining terms in Eq. (8) can still be significant (e.g., the term $-\frac{1}{2}\left[x_{0}^{1}\right]^{2} / D$ in this example), and (ii) this MFPT does not scale with the area of the domain. Interestingly, even though the area of the rectangle, $|\Omega|=L h$, stands in front of the logarithmic term in Eq. (8), the thickness $h$ is then removed by the factor $1 / h$ coming from $\ln \omega$. The last observation challenges another common paradigm that the MFPT is proportional to the area of the domain. In particular, if particles are released from a fixed point near the escape region, most of them find it very rapidly. Would the contribution to the MFPT from a few particles that miss the escape region at the beginning and thus explore the whole domain, ensure the scaling $\mathcal{T}\left(x_{0}\right) \propto|\Omega|$ ? The answer is in general negative as discussed in SM6 and illustrated above in the case of a thin rectangle.

We can now revise the second example mentioned above, namely, a disk with a small but highly corrugated arc. According to Makarov's theorem, the information dimension of the harmonic measure is equal to 1 for planar connected sets [36, 37], implying that $\omega_{x_{0}}(\Gamma)$ scales with the diameter of $\Gamma$ 38]. In other words, the harmonic measure of the corrugated arc, seen from a distant point $x_{0}$, is determined by the diameter $\delta$ of the arc, not the perimeter $\varepsilon$. We thus recover the intuitively expected behavior $\frac{|\Omega|}{\pi D} \ln (1 / \delta)$ when $\delta$ is small. In turn, the conventional formula $\frac{|\Omega|}{\pi D} \ln (1 / \varepsilon)$ is again strongly misleading. We emphasize that $\omega$ and $\varepsilon$ are in general unrelated; e.g., a set may have an arbitrarily small harmonic measure and arbitrarily large perimeter, and vice versa.

These examples illustrate generic features of the MFPT in porous media with long channels or fjords, and in domains with irregular boundaries, in contrast to earlier works that dealt with very regular domains whose perimeter $|\partial \Omega|$ and linear size $|\Omega|^{\frac{1}{2}}$ were comparable (see [5]). In the latter case, the harmonic measure of the escape region is proportional to its Lebesgue measure (i.e., the perimeter), recovering the conventional $\ln (1 / \varepsilon)$ behavior, the proportionality coefficient (the harmonic measure density) and the related dependence on the starting point $x_{0}$ being "hidden" in the $O(1)$ term. We stress that even for such regular domains, the dependence on the starting point can be strong and provide the dominant contribution to the MFPT, as illustrated in Fig. 1b,c. For instance, one can think of two pores connected by a very narrow channel. If the particle starts inside one pore while the escape region is located on the boundary of the other pore, the MFPT can be made arbitrarily large by controlling the channel width, even if the escape region remains large whereas the condition $|\partial \Omega| \sim|\Omega|^{\frac{1}{2}}$ is satisfied. Only the average of $\mathcal{T}\left(x_{0}\right)$ over the starting point $x_{0}$ might recover the $\ln (1 / \varepsilon)$ dependence in the leading term. However, in many applications, the source of particles and the escape region are well separated (e.g., viruses entering the cell at the membrane and searching for the nucleus, or molecules released near the nucleus and searching to escape through the membrane). The proposed formula (6) thus yields a powerful tool to investigate these search and escape phenomena.

The uncovered relation between the MFPT and the harmonic measure brings new opportunities. On one hand, one can profit from numerous analytical and numerical results known for the harmonic measure on irregular boundaries [22, 36, 37, 39 49]. In particular, the concept of diffusion screening [38, 50] that has found numerous implications for heterogeneous catalysis [51], fluid flow in rough channels 34, 52], and transport phenomena in biological systems [53 -56] can now be applied to the MFPT. For instance, the harmonic measure of an escape region at the bottom of a fjord can exhibit various types of decay with the "depth," depending on the shape [22, 39]. Similar dependences are thus expected for the MFPT. On the other hand, the conformal mapping is a powerful analytical and numerical technique to represent the geometric complexity of a domain through analytic properties of the mapping function $\phi_{x_{0}}(z)[57$ ]. The unit disk can be conformally mapped onto any polygon either by a Schwarz-Christoffel formula [58, 59] or by a "zipper" algorithm [60]. Once the conformal map is constructed, finding the MFPT in complex domains is reduced to computing the integrals in Eqs. (44) or (6). Most importantly, the proposed approach is not limited to regular domains (with $|\partial \Omega| \sim|\Omega|^{\frac{1}{2}}$ ) and allows one to study the MFPT for irregular (e.g., fractal) boundaries and branching domains with long rough channels and large surface-to-volume ratios that are relevant for most applications. This approach opens thus a new field of research on first passage times and related chemical or biochemical kinetics in complex, multiscale, and porous media.

\section{Acknowledgments}

The author acknowledges support under Grant No. ANR-13-JSV5-0006-01 of the French National Research Agency, and Dr. A. Rutenberg and Dr. D. Belyaev for fruitful discussions.
[1] J. W. S. Baron Rayleigh, The Theory of Sound, Vol. 2, 2nd Ed. (Dover, New York, 1945).
[2] S. Redner, A Guide to First Passage Processes (Cambridge, Cambridge University press, 2001). 
[3] R. Metzler, G. Oshanin, and S. Redner, First-Passage Phenomena and Their Applications (Singapore, World Scientific, 2014).

[4] O. Bénichou and R. Voituriez, Phys. Rep. 539, 225-284 (2014).

[5] D. Holcman and Z. Schuss, SIAM Rev. 56, 213-257 (2014).

[6] P. Hänggi, P. Talkner, and M. Borkovec, Rev. Mod. Phys. 62, 251-341 (1990).

[7] P. C. Bressloff and J. M. Newby, Rev. Mod. Phys. 85, 135-196 (2013).

[8] R. Zwanzig and A. Szabo, Biophys. J. 60, 671-678 (1991).

[9] I. V. Grigoriev, Y. A. Makhnovskii, A. M. Berezhkovskii, and V. Y. Zitserman, J. Chem. Phys. 116, 9574-9577 (2002).

[10] D. Holcman and Z. Schuss, J. Stat. Phys. 117, 975-1014 (2004).

[11] Z. Schuss, A. Singer, and D. Holcman, Proc. Nat. Acad. Sci. USA 104, 16098-16103 (2007).

[12] G. M. Viswanathan, S. V. Buldyrev, S. Havlin, M. G. E. da Luz, E. P. Raposok, and H. E. Stanley, Nature 401, 911-914 (1999).

[13] M. J. Ward and J. B. Keller, SIAM J. Appl. Math. 53, 770-798 (1993).

[14] A. Singer, Z. Schuss, D. Holcman, and R. S. Eisenberg, J. Stat. Phys. 122, 437-463 (2006).

[15] A. Singer, Z. Schuss, and D. Holcman, J. Stat. Phys. 122, 465-489 (2006).

[16] A. Singer, Z. Schuss, and D. Holcman, J. Stat. Phys. 122, 491-509 (2006).

[17] S. Pillay, M. J. Ward, A. Peirce, and T. Kolokolnikov, SIAM Multi. Model. Simul. 8, 803-835 (2010).

[18] A. F. Cheviakov, M. J. Ward, and R. Straube, SIAM Multi. Model. Simul. 8, 836-870 (2010).

[19] A. F. Cheviakov, A. S. Reimer, and M. J. Ward, Phys. Rev. E 85, 021131 (2012).

[20] C. Caginalp and X. Chen, Arch. Rational. Mech. Anal. 203, 329-342 (2012).

[21] J.-F. Rupprecht, O. Bénichou, D. S. Grebenkov, and R. Voituriez, J. Stat. Phys. 158, 192-230 (2015).

[22] J. B. Garnett and D. E. Marshall, Harmonic Measure (Cambridge, Cambridge University Press, 2005).

[23] C. W. Gardiner, Handbook of stochastic methods for physics, chemistry and the natural sciences (Berlin, Springer, 1985).

[24] I. N. Sneddon, Mixed Boundary Value Problems in Potential Theory (Wiley, NY, 1966).

[25] M. Brady and C. Pozrikidis, Proc. R. Soc. Lond A 442, 571-583 (1993).

[26] J. Koplik, S. Redner, and E. J. Hinch, Phys. Rev. E 50, 4650-4671 (1994).

[27] J. Koplik, S. Redner, and E. J. Hinch, Phys. Rev. Lett. 74, 82-85 (1995).

[28] M. B. Hastings and L. S. Levitov, Physica D 116, 244252 (1998).

[29] B. Davidovitch, H. G. E. Hentschel, Z. Olami, I. Procaccia, L. M. Sander, and E. Somfai, Phys. Rev. E 59, 1368-1378 (1999).

[30] M. G. Blyth and C. Pozrikidis, Int. J. Heat Mass Transf. 46, 1329-1339 (2003).

[31] M. Z. Bazant and D. Crowdy in the Handbook of Materials Modeling, ed. by S. Yip et al., Vol. I, Art. 4.10 (Springer, 2005).

[32] X. Chen and A. Friedman, SIAM J. Math. Anal. 43,
2542-2563 (2011)

[33] D. Holcman and Z. Schuss, Phys. Progr. Rep. 76, 074601 (2013).

[34] M. Z. Bazant, Phys. Rev. Fluids 1, 024001 (2016).

[35] A. Cherstvy, A. Chechkin, and R. Metzler, New J. Phys. 15, 083039 (2013).

[36] N. G. Makarov, Proc. London Math. Soc. 51, 369-384 (1985).

[37] P. W. Jones and T. H. Wolff, Acta Math. 161, 131-144 (1988)

[38] B. Sapoval, Phys. Rev. Lett. 73, 3314-3316 (1994).

[39] C. J. G. Evertsz, P. W. Jones, and B. Mandelbrot, J. Phys. A 24, 1889-1901 (1991).

[40] M. E. Cates and T. A. Witten, Phys. Rev. A 35, 18091824 (1987).

[41] P. Meakin, Phys. Rev. A 35, 2234-2245 (1987).

[42] B. B. Mandelbrot and C. J. G. Evertsz, Nature 348, 143145 (1990).

[43] C. J. G. Evertsz and B. B. Mandelbrot, J. Phys. A. 25, 1781-1797 (1992).

[44] N. G. Makarov, St. Petersburg Math. J. 10, 217-268 (1999).

[45] B. Duplantier, Phys. Rev. Lett. 82, 3940-3943 (1999).

[46] D. S. Grebenkov, A. A. Lebedev, M. Filoche, and B. Sapoval, Phys. Rev. E 71, 056121 (2005).

[47] E. Bettelheim, I. Rushkin, I. A. Gruzberg, and P. Wiegmann, Phys. Rev. Lett. 95, 170602 (2005).

[48] D. S. Grebenkov, Phys. Rev. E 91, 052108 (2015).

[49] D. S. Grebenkov Phys. Rev. Lett. 95, 200602 (2005).

[50] P. Meakin, H. E. Stanley, A. Coniglio, and T. A. Witten, Phys. Rev. A 32, 2364-2369 (1985).

[51] M. Filoche, D. S. Grebenkov, J. S. Andrade, and B. Sapoval, Proc. Nat. Acad. Sci. USA 105, 7636-7640 (2008).

[52] J. S. Andrade, A. D. Araújo, M. Filoche, and B. Sapoval, Phys. Rev. Lett. 98, 194101 (2007).

[53] M. Felici, M. Filoche, and B. Sapoval, J. Appl. Physiol. 94, 2010-2016 (2003).

[54] D. S. Grebenkov, M. Filoche, B. Sapoval, and M. Felici, Phys. Rev. Lett. 94, 050602 (2005).

[55] J. Gill, C. Salafia, D. S. Grebenkov, and D. Vvedensky, J. Theor. Biol. 291, 33-41 (2011).

[56] M. Mayo, S. Gheorghiu, and P. Pfeifer, Phys. Rev. E 85, 011115 (2012).

[57] R. Schinzinger and P. A. A. Laura, Conformal Mapping: Methods and Applications (Dover Publications, Mineola, New York, 2003).

[58] T. A. Driscoll and L. N. Trefethen, Schwarz-Christoffel Mapping (Cambridge Monographs on Applied and Computational Mathematics, 2002).

[59] L. Banjai and L. N. Trefethen, SIAM J. Sci. Comput. 25, 1042-1065 (2003).

[60] D. E. Marshall and S. Rohde, SIAM J. Num. Anal. 45, 2577-2609 (2007).

[61] I. S. Gradshteyn and I. M. Ryzhik, Table of Integrals, Series, and Products (Academic Press, 1980). 
Supplementary Material for the Letter "Universal formula for the mean first passage time in planar domains"

\section{SM1. DERIVATION OF THE MAIN FORMULA}

In this Section, we present the derivation of the exact formula for the MFPT $\mathcal{T}\left(x_{0}\right)$.

The function $\phi_{x_{0}}(z)$ that conformally maps the unit disk $\mathcal{D}$ onto the domain $\Omega$, transforms the original boundary value problem for the MFPT $\mathcal{T}\left(x_{0}\right)$ in $\Omega$,

$$
\left\{\begin{aligned}
\Delta \mathcal{T}\left(x_{0}\right) & =-1 / D\left(x_{0}\right) & & x_{0} \in \Omega, \\
\mathcal{T}\left(x_{0}\right) & =0 & & x_{0} \in \Gamma, \\
\partial_{n} \mathcal{T}\left(x_{0}\right) & =0 & & x_{0} \in \partial \Omega \backslash \Gamma,
\end{aligned}\right.
$$

into another boundary value problem for $\tau(z)=$ $\mathcal{T}\left(\phi_{x_{0}}(z)\right)$ in $\mathcal{D}$

$$
\left\{\begin{aligned}
\Delta \tau(z) & =f(z) & & z \in \mathcal{D}, \\
\tau(z) & =0 & & z \in \gamma, \\
\partial_{n} \tau(z) & =0 & & z \in \partial \mathcal{D} \backslash \gamma
\end{aligned}\right.
$$

where

$$
f(z)=-\frac{\left|\phi_{x_{0}}^{\prime}(z)\right|^{2}}{D\left(\phi_{x_{0}}(z)\right)},
$$

and the $\operatorname{arc} \gamma=(-\pi \omega, \pi \omega)$ is the pre-image of the escape region $\Gamma$. Here $0 \leq \omega \leq 1$ is the harmonic measure of the escape region seen from the starting point $x_{0}$ [22]. Since we imposed $\phi_{x_{0}}(0)=x_{0}$ for a fixed $x_{0}$, we have $\mathcal{T}\left(x_{0}\right)=$ $\tau(0)$ for this particular starting point. This choice of the conformal map greatly simplifies the following derivation.

The solution of the problem (S2) can be represented as $\tau(z)=u(z)+v(z)$, with $v(z)$ satisfying the inhomogeneous Laplace equation with Dirichlet boundary condition,

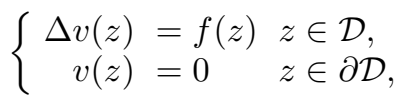

while $u(z)$ satisfying homogeneous Laplace equation with mixed Dirichlet-Neumann boundary condition:

$$
\left\{\begin{aligned}
\Delta u(z) & =0 & & z \in \mathcal{D}, \\
u(z) & =0 & & z \in \gamma, \\
\partial_{n} u(z) & =-\partial_{n} v(z) & & z \in \partial \mathcal{D} \backslash \gamma .
\end{aligned}\right.
$$

The solution of the problem (S4 is

$$
v(z)=\int_{\mathcal{D}} d z_{0} G\left(z, z_{0}\right) f\left(z_{0}\right),
$$

where $G\left(z, z_{0}\right)$ is the Green's function on the unit disk,

$$
G\left(z, z_{0}\right)=\frac{1}{2 \pi} \ln \left|\frac{z-z_{0}}{1-\bar{z}_{0} z}\right|,
$$

and bar denotes complex conjugate. In what follows, we focus on the solution of the homogeneous Laplace equation (S5).

In order to reduce this problem to dual trigonometric equations, we split the solution in "symmetric" and "antisymmetric" parts, $u=u_{+}+u_{-}$, which satisfy

$$
\left\{\begin{aligned}
\Delta u_{ \pm}(z) & =0 & & z \in \mathcal{D}, \\
u_{ \pm}(z) & =0 & & z \in \gamma, \\
\partial_{n} u_{ \pm}(z) & =-\frac{1}{2}\left[\partial_{n} v(z) \pm \partial_{n} v(\bar{z})\right] & & z \in \partial \mathcal{D} \backslash \gamma
\end{aligned}\right.
$$

In polar coordinates, $u_{+}(r, \theta)$ and $u_{-}(r, \theta)$ are respectively symmetric and antisymmetric functions with respect to reflection $\theta \rightarrow-\theta$.

We search the symmetric solution as

$$
u_{+}(r, \theta)=\frac{1}{2} a_{0}+\sum_{n=1}^{\infty} a_{n} r^{n} \cos n \theta,
$$

with unknown coefficients $a_{n}$ fixed by boundary conditions:

$$
\begin{array}{cc}
\left(\partial_{r} u_{+}(r, \theta)\right)_{r=1}=\sum_{n=1}^{\infty} n a_{n} \cos n \theta=F(\theta) & 0 \leq \theta \leq c, \\
u_{+}(1, \theta)=\frac{1}{2} a_{0}+\sum_{n=1}^{\infty} a_{n} \cos n \theta=0 & c \leq \theta \leq \pi,
\end{array}
$$

where

$$
\begin{gathered}
c=\pi-\pi \omega, \\
F(\theta)=-\frac{1}{2}\left[\partial_{r} v(r, \theta)+\partial_{r} v(r,-\theta)\right]_{r=1} \\
=-\frac{1}{2} \int_{\mathcal{D}} d z_{0}\left[\omega_{z_{0}}(\theta)+\omega_{z_{0}}(-\theta)\right] f\left(z_{0}\right),
\end{gathered}
$$

and

$$
\omega_{z_{0}}(\theta)=\left[\partial_{r} G\left(z, z_{0}\right)\right]_{r=1}=\frac{1-r_{0}^{2}}{2 \pi\left(1-2 r_{0} \cos \left(\theta-\theta_{0}\right)+r_{0}^{2}\right)}
$$

is the Poisson kernel (i.e., the harmonic measure density in the unit disk), with $z_{0}=r_{0} e^{i \theta_{0}}$. Note that we temporarily rotated the arc $\gamma$ to be $(c, 2 \pi-c)$ that is equivalent to replacing $\theta$ by $\pi-\theta$. We will rotate it back at the end of derivation.

The solution of the dual equations (S10) is given in 24] (see Eqs. (5.4.54, 5.4.55) with $G(\theta)=0$ and $\lambda=0$ ):

$$
\begin{aligned}
\frac{a_{0}}{2} & =\frac{\sqrt{2}}{\pi} \int_{0}^{c} d \theta \frac{\sin (\theta / 2)}{\sqrt{\cos \theta-\cos c}} \int_{0}^{\theta} d \theta^{\prime} F\left(\theta^{\prime}\right) \\
& =-\int_{\mathcal{D}} d z_{0} W_{\omega}\left(z_{0}\right) f\left(z_{0}\right),
\end{aligned}
$$


with

$$
\begin{aligned}
W_{\omega}\left(z_{0}\right) & =\frac{1}{\pi \sqrt{2}} \int_{0}^{c} d \theta \frac{\sin (\theta / 2)}{\sqrt{\cos \theta-\cos c}} \\
& \times \int_{0}^{\theta} d \theta^{\prime}\left[\omega_{z_{0}}\left(\theta^{\prime}\right)+\omega_{z_{0}}\left(-\theta^{\prime}\right)\right] .
\end{aligned}
$$

One can also get the other coefficients $a_{n}$ but they are not needed since we are only interested in $u_{+}(0,0)$.

In order to get an explicit representation of $W_{\omega}\left(z_{0}\right)$, we rewrite the Poisson kernel in Eq. (S12) as

$$
\omega_{z_{0}}(\theta)=\frac{1}{2 \pi}\left[1+2 \sum_{n=1}^{\infty} r_{0}^{n} \cos n\left(\theta-\theta_{0}\right)\right]
$$

so that

$$
\frac{1}{2}\left[\omega_{z_{0}}(\theta)+\omega_{z_{0}}(-\theta)\right]=\frac{1}{2 \pi}\left[1+2 \sum_{n=1}^{\infty} r_{0}^{n} \cos n \theta \cos n \theta_{0}\right] .
$$

Integration of this function with respect to $\theta$ yields

$$
\begin{aligned}
W_{\omega}\left(z_{0}\right) & =\frac{1}{\pi^{2} \sqrt{2}} \int_{0}^{c} d \theta \frac{\sin (\theta / 2)}{\sqrt{\cos \theta-\cos c}} \\
& \times\left[\theta+2 \sum_{n=1}^{\infty} \frac{r_{0}^{n}}{n} \cos n \theta_{0} \sin n \theta\right] .
\end{aligned}
$$

To proceed, we will use the identity (see [24], p. 59, 2.6.30)

$$
\frac{\sin (\theta / 2) \Theta(c-\theta)}{\sqrt{\cos \theta-\cos c}}=\frac{1}{\sqrt{2}} \sum_{k=1}^{\infty}\left(P_{k-1}(\cos c)-P_{k}(\cos c)\right) \sin k \theta,
$$

where $\Theta(c-\theta)$ is the Heaviside step function. Multiplying this identity by $\theta$ and integrating over $\theta$ from 0 to $\pi$, we get

$$
\begin{aligned}
& \int_{0}^{c} d \theta \frac{\sin (\theta / 2)}{\sqrt{\cos \theta-\cos c}} \theta \\
& =\frac{\pi}{\sqrt{2}} \sum_{k=1}^{\infty}\left(P_{k-1}(\cos c)-P_{k}(\cos c)\right) \frac{(-1)^{k+1}}{k} \\
& =-\pi \sqrt{2} \ln \cos (c / 2) .
\end{aligned}
$$

To prove the last relation, one can decompose $\ln (1+x)$ (with $x=\cos c$ ) on Legendre polynomials as

$$
\begin{aligned}
\ln (1+x) & =\ln 2-1+\sum_{n=1}^{\infty}(-1)^{n+1} P_{n}(x) \frac{2 n+1}{n(n+1)} \\
& =\ln 2+\sum_{n=1}^{\infty}(-1)^{n+1} \frac{P_{n}(x)-P_{n-1}(x)}{n} .
\end{aligned}
$$

Similarly, one multiplies Eq. (S19) by $\sin n \theta$ and integrates from 0 to $\pi$ to get

$$
\int_{0}^{c} d \theta \frac{\sin (\theta / 2)}{\sqrt{\cos \theta-\cos c}} \sin n \theta=\frac{\pi\left(P_{n-1}(\cos c)-P_{n}(\cos c)\right)}{2 \sqrt{2}} .
$$

Combining these results, we find

$$
\begin{aligned}
W_{\omega}\left(z_{0}\right) & =-\frac{\ln \cos (c / 2)}{\pi} \\
& +\frac{1}{2 \pi} \sum_{n=1}^{\infty} \frac{P_{n-1}(\cos c)-P_{n}(\cos c)}{n} r_{0}^{n} \cos n \theta_{0} .
\end{aligned}
$$

Using Eq. (S11) and $P_{n}(-x)=(-1)^{n} P_{n}(x)$, we obtain

$$
\begin{aligned}
W_{\omega}\left(z_{0}\right) & =-\frac{\ln \sin (\pi \omega / 2)}{\pi} \\
& -\sum_{n=1}^{\infty} \frac{P_{n-1}(\cos \pi \omega)+P_{n}(\cos \pi \omega)}{2 \pi n} r_{0}^{n} \cos n \theta_{0},
\end{aligned}
$$

where the arc was rotated back by replacing $\theta_{0}$ by $\pi-\theta_{0}$. Using the following identity (that we will prove independently in SM7)

$$
\begin{aligned}
& \sum_{n=1}^{\infty} \frac{P_{n}(\cos \epsilon)+P_{n-1}(\cos \epsilon)}{2 n} r^{n} \cos n \theta \\
& =-\ln \left(\frac{\mid 1-r e^{i \theta}+\sqrt{\left(1-r e^{i(\theta-\epsilon)}\right)\left(1-r e^{i(\theta+\epsilon)}\right) \mid}}{2}\right)
\end{aligned}
$$

we find another representation

$W_{\omega}\left(z_{0}\right)=\frac{1}{\pi} \ln \left(\frac{\left|1-z_{0}+\sqrt{\left(1-z_{0} e^{i \pi \omega}\right)\left(1-z_{0} e^{-i \pi \omega}\right)}\right|}{2 \sin (\pi \omega / 2)}\right)$.

The behavior of this function is illustrated in Fig. S1.

Similarly, one can find the antisymmetric solution by using an expansion over sine functions:

$$
u_{-}(r, \theta)=\sum_{n=1}^{\infty} a_{n}^{\prime} r^{n} \sin n \theta
$$

with unknown coefficients $a_{n}^{\prime}$ satisfying dual trigonometric equations obtained from the boundary conditions. However, since we are only interested in $u(0,0)$, there is no need to solve this equation.

Gathering the above results, we finally get

$$
\begin{aligned}
\mathcal{T}\left(x_{0}\right) & =\tau(0)=v(0)+u_{+}(0,0)+u_{-}(0,0) \\
& =\int_{\mathcal{D}} \frac{d z\left|\phi_{x_{0}}^{\prime}(z)\right|^{2}}{D\left(\phi_{x_{0}}(z)\right)}\left(-\frac{\ln |z|}{2 \pi}+W_{\omega}(z)\right),
\end{aligned}
$$



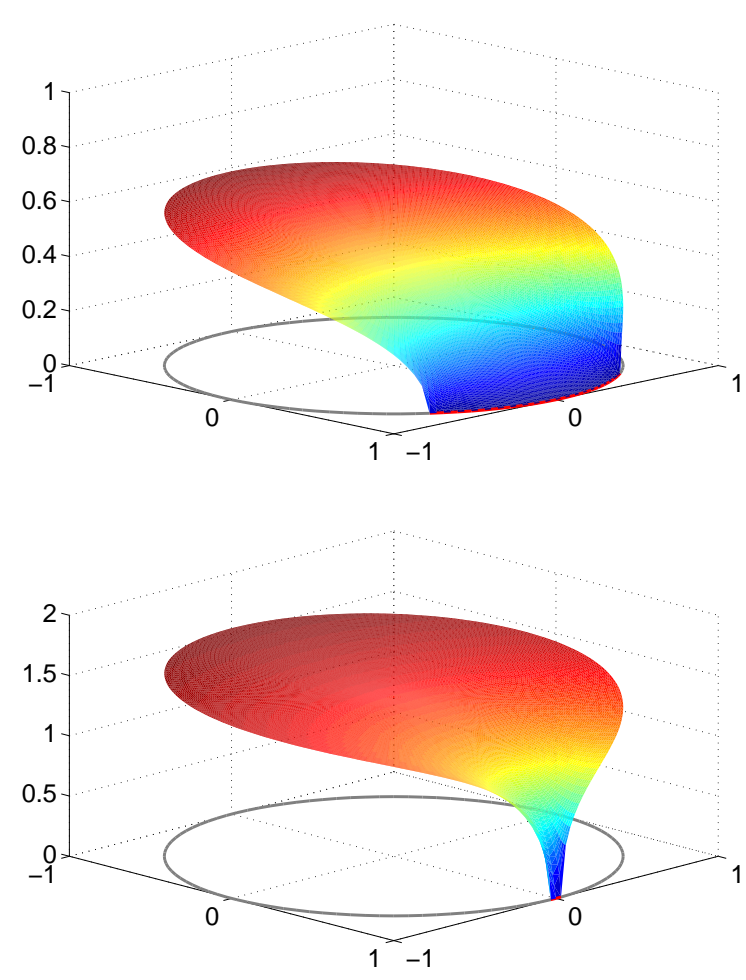

FIG. S1: The function $W_{\omega}(z)$ for $\omega=0.2$ (top) and $\omega=0.01$ (bottom). It vanishes on the arc $(-\pi \omega, \pi \omega)$.

from which the change of the integration variable $z$ to $x=\phi_{x_{0}}(z)$ yields

$$
\mathcal{T}\left(x_{0}\right)=\int_{\Omega} \frac{d x}{D(x)}\left(-\frac{\ln \left|\phi_{x_{0}}^{-1}(x)\right|}{2 \pi}+W_{\omega}\left(\phi_{x_{0}}^{-1}(x)\right)\right) .
$$

Note that the factor $\left|\phi_{x_{0}}^{\prime}(z)\right|^{2}$, which stands in Eq. (S27) is the Jacobian of this change of variables: $d x=$ $d z\left|\phi_{x_{0}}^{\prime}(z)\right|^{2}$.

In the formula (S28), the first integral that we denote as

$$
\mathcal{T}_{D}\left(x_{0}\right)=-\int_{\Omega} \frac{d x}{D(x)} \frac{\ln \left|\phi_{x_{0}}^{-1}(x)\right|}{2 \pi},
$$

is the MFPT to the whole boundary, whereas the second term accounts for eventual reflections on the remaining part of the boundary, $\partial \Omega \backslash \Gamma$, until reaching the escape region $\Gamma$.

We recall that the harmonic measure $\omega=\omega_{x_{0}}(\Gamma)$ depends on the starting point $x_{0}$ and solves the following Dirichlet boundary value problem in $\Omega$ :

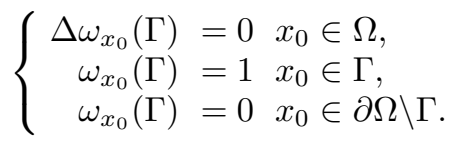

It can also be expressed in terms of the Green's function, or through the conformal map by noting that the endpoints $x_{1}$ and $x_{2}$ of the escape region $\Gamma$ (enumerated in the counterclockwise order) are mapped back onto the points $e^{-i \pi \omega}$ and $e^{i \pi \omega}$ of the circle of the unit disk. In general, one has

$$
x_{1}=\phi_{x_{0}}\left(e^{i \alpha-i \pi \omega}\right), \quad x_{2}=\phi_{x_{0}}\left(e^{i \alpha+i \pi \omega}\right),
$$

where the factor $e^{i \alpha}$ aims at rotating the unit disk to put the pre-image $\gamma$ onto the arc $(-\pi \omega, \pi \omega)$. One gets therefore

$$
\begin{aligned}
e^{2 i \pi \omega} & =\phi_{x_{0}}^{-1}\left(x_{1}\right) / \phi_{x_{0}}^{-1}\left(x_{2}\right), \\
e^{2 i \alpha} & =\phi_{x_{0}}^{-1}\left(x_{1}\right) \phi_{x_{0}}^{-1}\left(x_{2}\right),
\end{aligned}
$$

from which both $\omega$ and $\alpha$ can be determined.

Although the MFPT and the harmonic measure are both formulated as boundary value problems for the Laplace operator, their relation is not so intuitive. In fact, the harmonic measure $\omega=\omega_{x_{0}}(\Gamma)$ is the probability that the first arrival of Brownian motion on the boundary occurs at the escape region $\Gamma$. In other words, the harmonic measure characterizes the "accessibility" of the escape region $\Gamma$ in "competition" with the remaining part of the boundary $\partial \Omega \backslash \Gamma[49 \mid$. This competition is known as diffusion screening [38, 50], the concept that has found numerous implications for heterogeneous catalysis [51], fluid flow in rough channels 34,52 and transport phenomena in biological systems 53 56]. In turn, only the escape region is absorbing in the MFPT problem while the remaining boundary is reflecting. In particular, after hitting the reflecting boundary, the particle bounces back into the domain and continues to diffuse. The statistics of Brownian trajectories in these two settings are thus very different, that makes the relation (S28) particularly intricate.

At the end of this section, we outline the main mathematical facts that we used for derivation: (i) the infinitesimal generator of the Brownian motion is the Laplace operator; (ii) there exists a conformal mapping from the unit disk to any simply connected planar domain (Riemann's theorem); (iii) the conformal map preserves angles; and (iv) the Poisson equation with mixed DirichletNeumann boundary condition can be analytically solved in the disk. These facts highlight the intrinsic orientation of the proposed approach to planar Brownian motion. In particular, extensions to other stochastic processes (governed by a general elliptic or Fokker-Planck operator) or to higher dimensions remain unknown.

\section{SM2. NUMERICAL IMPLEMENTATION AND VALIDATION}

Practical implementation of the exact formula (S28) involves two numerical steps: computation of the conformal map and integration. 
(i) For a given polygonal domain, the first step can be realized either by a Schwarz-Christoffel formula [58], or by a "zipper" algorithm [60]. Once a conformal map $\phi_{x_{0}}(z)$ is constructed for a starting point $x_{0}$, the Möbius transform,

$$
M_{z_{0}}(z)=\frac{z_{0}-z}{1-\bar{z}_{0} z}
$$

yields the conformal map $\phi_{x_{0}^{\prime}}(z)$ for another starting point $x_{0}^{\prime}$ :

$$
\phi_{x_{0}^{\prime}}(z)=\phi_{x_{0}}\left(M_{\phi_{x_{0}}^{-1}\left(x_{0}^{\prime}\right)}(z)\right) .
$$

In other words, the numerical construction of the conformal map is needed only for one starting point. Note that the transformation (S34) can also be helpful to investigate the dependence of the MFPT on the starting point.

(ii) The numerical integration involves meshing of the domain and quadratures. The MFPT can be computed through either of two equivalent representations S27. S28). While the integration over the unit disk in Eq. (S27) facilitates meshing, it requires an accurate numerical treatment of integrable singularities of the derivative of the conformal map near pre-vertices (the factor $\left|\phi_{x_{0}}^{\prime}(z)\right|^{2}$. For this reason, we use the other option, in which the domain $\Omega$ is meshed by triangles, the integrand function in Eq. (S28) is evaluated at the vertices of these triangles and then summed to approximate the integral. We checked the accuracy of computation by doubling the number of triangles.

Although both numerical steps are well documented and controlled, it is instructive to illustrate their accuracy on two simple domains: disk and rectangle.

\section{A. Disk}

We first consider the MFPT through the escape arc $(\pi-\epsilon, \pi+\epsilon)$ on the boundary of the unit disk with a linearly varying diffusion coefficient along the radial coordinate: $D\left(x_{0}\right)=D\left(1+\eta\left|x_{0}\right|\right)$, with a gradient $\eta>-1$. In the special case $\eta=0$, one recovers the uniform diffusion coefficient, for which the explicit formula (S40) can be used (see SM3A for details). For other cases $(\eta \neq 0)$, we resort to a numerical solution of the original mixed boundary value problem (S1) by a finite element method (FEM) implemented in the Matlab PDE toolbox. Our universal formula (S28) is compared to a FEM numerical solution in Fig. S2 To control the quality of the FEM solution, we provide the numerical results for two mesh sizes. One observes an excellent agreement between the universal formula and both numerical solutions. Since the conformal map is trivial for this domain, the numerical implementation of the universal formula is much faster and much more accurate than that of a FEM.

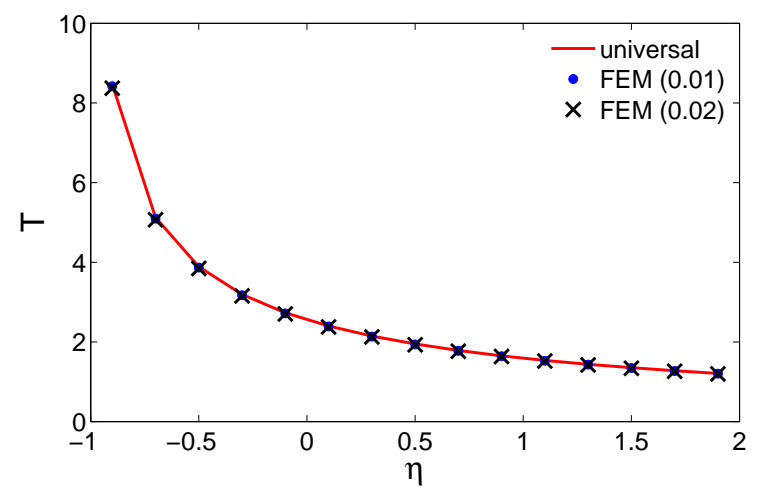

FIG. S2: The MFPT $\mathcal{T}(0)$ through the escape arc $(\pi-\epsilon, \pi+\epsilon)$ (with $\epsilon=0.2$ ) on the boundary of the unit disk as a function of the gradient $\eta$ of the linearly varying diffusion coefficient along the radial coordinate: $D\left(x_{0}\right)=1+\eta\left|x_{0}\right|$. The universal formula (S28 solid line) is compared to FEM solutions of Eqs. (S1) with two mesh sizes: 0.01 (circles) and 0.02 (crosses).

\section{B. Rectangle}

We next consider the MFPT to the left edge of the rectangle $[0, L] \times[0, h]$ with the diffusion coefficient $D\left(x_{0}\right)$ which depends only on the horizontal coordinate $x_{0}^{1}$. In this particular setting, the MFPT does not depend on the vertical coordinate $x_{0}^{2}$, and the remaining onedimensional problem can be solved exactly:

$$
\mathcal{T}\left(x_{0}\right)=\int_{0}^{L} d x^{1} \frac{G_{1}\left(x_{0}^{1}, x^{1}\right)}{D\left(x^{1}\right)},
$$

where $G_{1}\left(x_{0}^{1}, x^{1}\right)$ is the Green function for the interval $[0, L]$ with Dirichlet and Neumann boundary conditions at endpoints 0 and $L$, respectively:

$$
G_{1}\left(x_{0}^{1}, x^{1}\right)=2 L \sum_{n=0}^{\infty} \frac{\sin \left(\pi\left(n+\frac{1}{2}\right) \frac{x_{0}^{1}}{L}\right) \sin \left(\pi\left(n+\frac{1}{2}\right) \frac{x^{1}}{L}\right)}{\pi^{2}\left(n+\frac{1}{2}\right)^{2}} .
$$

For illustrative purposes, we choose a particular spatial dependence

$$
D\left(x^{1}\right)=\frac{D}{1+\beta \sin \left(\pi\left(m+\frac{1}{2}\right) \frac{x^{1}}{L}\right)}
$$

in order to get a simple explicit solution:

$$
\mathcal{T}\left(x_{0}\right)=\frac{L x_{0}^{1}-\frac{1}{2}\left(x_{0}^{1}\right)^{2}}{D}+\frac{\beta L^{2}}{D} \frac{\sin \left(\pi\left(m+\frac{1}{2}\right) \frac{x_{0}^{1}}{L}\right)}{\pi^{2}\left(m+\frac{1}{2}\right)^{2}},
$$

where $m$ is an integer and $|\beta|<1$. Here the first term is the MFPT that would be obtained for a constant diffusivity, while the second term results from periodic fluctuations of the chosen spatial dependence in $D\left(x^{1}\right)$. Figure 


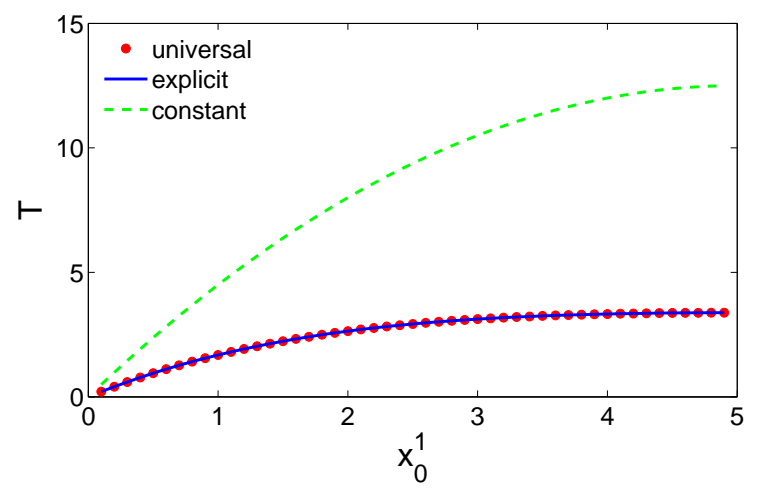

FIG. S3: The MFPT $\mathcal{T}\left(x_{0}\right)$ through the left edge of the rectangle $[0, L] \times[0, h]$ for space-dependent diffusion coefficient $D\left(x_{0}\right)$ in Eq. (S37), with $L=5, h=1, x_{0}^{2}=0.5, \beta=-0.9$, $m=0$, and $D=1$. The universal formula (S28 circles) is compared to the explicit solution (S38 solid line) available exclusively for this setting. Dashed line shows the first term, $\left(L x_{0}^{1}-\frac{1}{2}\left(x_{0}^{1}\right)^{2}\right) / D$, corresponding to the constant diffusion coefficient.

S3 illustrates the high accuracy of the numerical computation by the universal formula (S28). Although this example may look too simplistic, numerical computation of conformal maps is known to be challenging for elongated shapes because of the crowding phenomenon [58]. In spite of this potential difficulty, the maximal relative error of our numerical implementation of the universal formula in this example is below $0.003 \%$.

\section{Comparison to other numerical techniques}

Since practical implementations of the exact solution (S28) involve numerical steps, one may wonder how efficient this approach is in comparison to conventional numerical techniques for solving the boundary value problem (S1) such as finite element or finite difference methods or Monte Carlo simulations [19]. Although a systematic comparison between different techniques is beyond the scope of this letter, we outline one of the major advantages of the present approach from the numerical point of view. In general, conventional numerical techniques suffer in the narrow escape limit. Indeed, both finite element and finite difference methods would require very fine meshes to accurately treat the mixed boundary condition near a small escape region. Similarly, Monte Carlo simulations would be slowed down as longer trajectories need to be generated to access larger FPTs, while the number of these trajectories has be increased to compensate for higher dispersion of FPTs in the narrow escape limit. In contrast, the numerical implementation of the exact solution (S28) is expected to be less sensitive to the escape region size: (i) the computation of the conformal map is independent of boundary conditions and of the escape region size, and (ii) numerical integration of a smooth integrand function in Eq. (S28) does not require very fine meshes and can be further improved by using high order quadratures. In other words, the numerical advantage of the proposed approach results from the natural "representation" of a confining domain by the conformal map and from the incorporation of the mixed boundary condition through the explicit function $W_{\omega}(z)$. Moreover, the narrow escape limit is particularly favorable for the present approach since the asymptotic formula (S63) from SM4 becomes very accurate so that the leading logarithmic and constant terms can be enough for an accurate evaluation of the MFPT.

\section{SM3. ANALYTICAL RESULTS FOR DISK AND RECTANGLE}

To illustrate the use of the main formula (S28), we consider the MFPT in two basic domains, disk and rectangle, with a constant diffusion coefficient.

\section{A. Disk}

For the unit disk with an escape $\operatorname{arc}(-\epsilon, \epsilon)$, Singer et al. provided the exact solution for arbitrary $\epsilon$ in terms of an infinite series with coefficients in the form of integrals [15] that were later reduced to Legendre polynomials [21]:

$$
\begin{aligned}
\mathcal{T}\left(x_{0}\right) & =\frac{1}{D}\left(\frac{1-r_{0}^{2}}{4}-\ln \sin (\epsilon / 2)\right. \\
& \left.-\sum_{n=1}^{\infty} \frac{P_{n}(\cos \epsilon)+P_{n-1}(\cos \epsilon)}{2 n} r_{0}^{n} \cos n \theta_{0}\right),
\end{aligned}
$$

with $x_{0}=r_{0} e^{i \theta_{0}}$. A simpler explicit formula was obtained by Caginalp and Chen [20]:

$$
\begin{aligned}
\mathcal{T}\left(x_{0}\right) & =\frac{1}{D}\left[\frac{1-r_{0}^{2}}{4}\right. \\
& \left.+\ln \left(\frac{\left|1-x_{0}+\sqrt{\left(1-x_{0} e^{-i \epsilon}\right)\left(1-x_{0} e^{i \epsilon}\right)}\right|}{2 \sin (\epsilon / 2)}\right)\right] .
\end{aligned}
$$

The comparison of these two relations implies the identity (S24) that we derive independently in SM7.

Although the solution for the disk is known, it is instructive to recover it from the general formula (S27). Conformal mapping of the unit disk $\mathcal{D}$ onto itself is realized by a family of linear fractional transformations

$$
\phi(z)=e^{i \alpha} \frac{z-w}{1-\bar{w} z},
$$

where $\alpha$ is a real number and $w$ is a point in $\mathcal{D}$. Imposing the mapping of the origin of the disk onto $x_{0} \in \Omega(=\mathcal{D})$, we set

$$
\phi_{x_{0}}(z)=\frac{x_{0}-z e^{i \alpha}}{1-z \bar{x}_{0} e^{i \alpha}}
$$


where $\alpha$ is a real parameter which determines an appropriate rotation of the disk (in $z$ coordinates) to ensure the symmetry of the escape arc, $(-\pi \omega, \pi \omega)$. One gets thus

$$
\left|\phi_{x_{0}}^{\prime}(z)\right|=\frac{1-\left|x_{0}\right|^{2}}{1-z \bar{x}_{0} e^{i \alpha}-\bar{z} x_{0} e^{-i \alpha}+|z|^{2}\left|x_{0}\right|^{2}},
$$

while the inverse mapping is

$$
\phi_{x_{0}}^{-1}(x)=\frac{x_{0}-x}{1-x \bar{x}_{0}} e^{-i \alpha} .
$$

Relating the escape region $\Gamma=(-\epsilon, \epsilon)$ and its preimage $\gamma=(-\pi \omega, \pi \omega)$ by the conformal map (S42),

$$
e^{i \epsilon}=\phi_{x_{0}}\left(e^{i \pi \omega}\right), \quad e^{-i \epsilon}=\phi_{x_{0}}\left(e^{-i \pi \omega}\right),
$$

one finds $\omega$ and $\alpha$

$$
\begin{aligned}
& \omega=-\frac{i}{2 \pi} \ln \left(\frac{e^{i \epsilon}-x_{0}}{\bar{x}_{0} e^{i \epsilon}-1} \frac{\bar{x}_{0} e^{-i \epsilon}-1}{e^{-i \epsilon}-x_{0}}\right), \\
& \alpha=-i \ln \left(-\sqrt{\frac{e^{i \epsilon}-x_{0}}{\bar{x}_{0} e^{i \epsilon}-1} \frac{e^{-i \epsilon}-x_{0}}{\bar{x}_{0} e^{-i \epsilon}-1}}\right) .
\end{aligned}
$$

These relations can also be re-written as

$$
\omega=\omega_{x_{0}}(\Gamma)= \begin{cases}\frac{1}{\pi} \arctan (\eta) & (\eta>0), \\ 1+\frac{1}{\pi} \arctan (\eta) & (\eta<0),\end{cases}
$$

with

$$
\eta=\frac{\left(1-r_{0}^{2}\right) \sin \epsilon}{\left(1+r_{0}^{2}\right) \cos \epsilon-2 r_{0} \cos \theta_{0}}
$$

and

$$
\alpha=\arctan \left(\frac{2 r_{0} \sin \theta_{0}\left(r_{0} \cos \theta_{0}-\cos \epsilon\right)}{1-r_{0}^{2}+2 r_{0} \cos \theta_{0}\left(r_{0} \cos \theta_{0}-\cos \epsilon\right)}\right) .
$$

Note that the harmonic measure $\omega$ could alternatively be determined by integrating the Poisson kernel (S13).

Substituting (S44) into the first term of Eq. (S27) and computing the integral in polar coordinates, one retrieves the classical MFPT to the unit circle:

$$
\mathcal{T}_{D}\left(x_{0}\right)=\frac{1-r_{0}^{2}}{4 D}
$$

where we used the identity (see [61], p. 541)

$$
\int_{0}^{2 \pi} d \theta \ln \left(1+2 r \cos \theta+r^{2}\right)=0 \quad \text { for }|r| \leq 1 .
$$

Using the series representation (S23), one computes the second contribution in Eq. (S27) by integrating term by term

$$
\begin{aligned}
& \int_{\mathcal{D}} \frac{d z}{D} W_{\omega}(z)\left|\phi_{x_{0}}^{\prime}(z)\right|^{2}=\frac{-1}{2 \pi D} \sum_{n=1}^{\infty} c_{n} \\
& \times \int_{0}^{1} d r r \int_{0}^{2 \pi} d \theta \frac{\left(1-r_{0}^{2}\right)^{2} r^{n} \cos n \theta}{\left(1-2 r r_{0} \cos \left(\theta-\theta_{0}+\alpha\right)+r_{0}^{2} r^{2}\right)^{2}} \\
& =\frac{-1}{D} \sum_{n=1}^{\infty} c_{n} r_{0}^{n} \cos n\left(\theta_{0}-\alpha\right),
\end{aligned}
$$

with

$$
c_{n}=\frac{P_{n-1}(\cos \pi \omega)+P_{n}(\cos \pi \omega)}{2 n},
$$

where we used the identity

$$
\int_{0}^{1} d r \int_{0}^{2 \pi} \frac{r^{n+1} \cos n \theta d \theta}{\left(1-2 a r \cos \left(\theta-\theta_{0}\right)+a^{2} r^{2}\right)^{2}}=\frac{\pi a^{n} \cos n \theta_{0}}{\left(1-a^{2}\right)^{2}} .
$$

Combining these results, one gets

$$
\begin{aligned}
\mathcal{T}\left(x_{0}\right) & =\frac{1}{D}\left[\frac{1-r_{0}^{2}}{4}-\frac{1}{\pi} \ln \sin (\pi \omega / 2)-\sum_{n=1}^{\infty} \frac{P_{n-1}(\cos \pi \omega)+P_{n}(\cos \pi \omega)}{2 n} r_{0}^{n} \cos n\left(\theta_{0}-\alpha\right)\right] \\
& =\frac{1}{D}\left[\frac{1-r_{0}^{2}}{4}+\ln \left(\frac{\mid 1-r_{0} e^{i\left(\theta_{0}-\alpha\right)}+\sqrt{\left(1-r_{0} e^{i\left(\theta_{0}-\alpha+\pi \omega\right)}\right)\left(1-r_{0} e^{i\left(\theta_{0}-\alpha-\pi \omega\right)}\right)}}{2 \sin (\pi \omega / 2)}\right)\right]
\end{aligned}
$$

where we used the identity (S24). Comparing Eqs. (S40, S53), one gets the following relation for the unit disk:

$$
W_{\varepsilon}\left(x_{0}\right)=W_{\omega}\left(x_{0} e^{-i \alpha}\right),
$$

where $\omega$ and $\alpha$ are related to $x_{0}$ according to Eqs. $\mathbf{S 4 7}$ [49), and $\varepsilon=|\Gamma| /|\partial \Omega|=\epsilon / \pi$.

\section{B. Thin rectangle}

We consider now the MFPT from a thin rectangle $\Omega=$ $[0, L] \times[0, h]$ through its left edge: $\Gamma=\{0\} \times[0, h]$. The 
exact solution of this problem is simply

$$
\mathcal{T}\left(x_{0}\right)=\frac{2 L x_{0}^{1}-\left[x_{0}^{1}\right]^{2}}{2 D},
$$

which does not depend on $x_{0}^{2}$ and $h$ (in this subsection, we use the Cartesian coordinates, $x_{0}=\left(x_{0}^{1}, x_{0}^{2}\right)$, instead of polar coordinates or complex numbers). One can see that the MFPT is not determined by the normalized perimeter $\varepsilon=\frac{h}{2(L+h)}$, even if the latter is very small.

The harmonic measure $\omega_{x_{0}}(\Gamma)$ is obtained by solving Eq. (S30):

$\omega_{x_{0}}=\sum_{n=1}^{\infty} \frac{2\left(1-(-1)^{n}\right)}{\pi n} \sin \left(\pi n x_{0}^{2} / h\right) \frac{\sinh \left(\pi n\left(L-x_{0}^{1}\right) / h\right)}{\sinh (\pi n L / h)}$

Setting the starting point on the horizontal line at the middle, $x_{0}^{2}=h / 2$, and omitting exponentially small terms with $n=2,3, \ldots$ (for $h \ll L)$, one gets $\omega_{\left(x_{0}^{1}, h / 2\right)} \simeq$ $\frac{4}{\pi} e^{-\pi x_{0}^{1} / h}$, from which Eq. (S63) yields the leading term

$$
\mathcal{T}\left(x_{0}^{1}, h / 2\right) \simeq-\frac{L h}{\pi D} \ln \left(\omega_{\left(x_{0}^{1}, h / 2\right)}\right) \simeq \frac{L x_{0}^{1}}{D},
$$

in agreement with the exact solution (S55). The missing term $-\frac{1}{2}\left[x_{0}^{1}\right]^{2} / D$ from Eq. (S55) is related to the presence of the reflecting edge at $x^{1}=L$ which is accounted for by the remaining terms in Eq. (S63). If the starting point $x_{0}$ was located near a long edge (i.e., if $x_{0}^{2}$ was close to 0 or $h$ ), one would get an extra term, $-\frac{L h}{\pi D} \ln \sin \left(\pi x_{0}^{2} / h\right)$ but its contribution would be compensated by the remaining terms in Eq. S63 that we ignored here. Note that the MFPT to the whole boundary, $\mathcal{T}_{D}\left(x_{0}\right)$, can be computed exactly but it vanishes as $h \rightarrow 0$.

Now we consider the MFPT from the same rectangle but through any edge except the right one. Here the Poisson equation, $\Delta \mathcal{T}\left(x_{0}\right)=-1 / D$, is completed by boundary conditions: $\mathcal{T}\left(x_{0}^{1}, 0\right)=\mathcal{T}\left(x_{0}^{1}, h\right)=\mathcal{T}\left(0, x_{0}^{2}\right)=0$ (escape region) and $\partial \mathcal{T} / \partial x_{0}^{1}=0$ at $x_{0}^{1}=L$ (reflecting region). One gets an explicit solution

$$
\begin{gathered}
\mathcal{T}\left(x_{0}\right)=\frac{x_{0}^{2}\left(h-x_{0}^{2}\right)}{2 D}-\frac{2 h^{2}}{D} \sum_{n=1}^{\infty} \frac{1-(-1)^{n}}{\pi^{3} n^{3}} \quad \text { (S58) where } \\
\quad \times \sin \left(\pi n x_{0}^{2} / h\right) \frac{\cosh \left(\pi n\left(L-x_{0}^{1}\right) / h\right)}{\cosh (\pi n I h)} . \\
V_{0}(z)=-\frac{1}{2 \pi} \sum_{n=1}^{\infty} \frac{2}{n} r^{n} \cos n \theta=\frac{1}{2 \pi} \ln \left(1-2 r \cos \theta+r^{2}\right)=\frac{1}{2 \pi} \ln ((1-z)(1-\bar{z}))=\frac{1}{\pi} \ln |1-z|, \\
V_{2}(z)=-\frac{\pi}{4} \sum_{n=1}^{\infty} n r^{n} \cos n \theta=\frac{\pi r}{4} \frac{2 r-\left(1+r^{2}\right) \cos \theta}{\left(1-2 r \cos \theta+r^{2}\right)^{2}}=-\frac{\pi}{8}\left[\frac{z}{(1-z)^{2}}+\frac{\bar{z}}{(1-\bar{z})^{2}}\right]=\frac{\pi}{8}\left(\frac{\left(1+|z|^{2}\right)}{|1-z|^{2}}-\frac{\left(1-|z|^{2}\right)^{2}}{|1-z|^{4}}\right) .
\end{gathered}
$$

When the starting point $x_{0}$ is not close to the left or right edges (i.e., $h \ll x_{0}^{1}$ and $h \ll L-x_{0}^{1}$ ), the contribution from the second term is exponentially small (of the order of $e^{-\pi x_{0}^{1} / h}$ ), and the MFPT is determined by the first term (describing the one-dimensional problem along the vertical coordinate). In the limit $x_{0}^{1} \rightarrow 0$, one gets (for $L \gg h)$

$$
\mathcal{T}\left(x_{0}\right) \simeq x_{0}^{1} \frac{2 h}{D} \sum_{n=1}^{\infty} \frac{1-(-1)^{n}}{\pi^{2} n^{2}} \sin \left(\pi n x_{0}^{2} / h\right) .
$$

For instance, setting $x_{0}^{2}=h / 2$, the sum is evaluated numerically, yielding $\mathcal{T}\left(x_{0}\right) \simeq 0.37 x_{0}^{1} h / D$. This relation is similar to Eq. (S57), with the length $L$ of the rectangle being replaced by its width $h$. In both relations (S57, S59), the MFPT does not scale with the area of the domain. Moreover, in the latter case, one can take the limit $L \rightarrow \infty$ and consider an infinite half-stripe (of infinite area) for which the MFPT remains finite.

\section{SM4. ASYMPTOTIC BEHAVIOR}

When the escape region $\Gamma$ is the whole boundary (no reflecting part), the harmonic measure $\omega$ is equal 1 , the function $W_{\omega}(z)$ vanishes, and one recovers the conventional solution $\mathcal{T}_{D}\left(x_{0}\right)$ for the Dirichlet problem, as expected.

In this Section, we focus on the more interesting limit $\omega \rightarrow 0$. Using

$$
P_{n}(\cos (\pi \omega))=1-\frac{n(n+1)}{4} \pi^{2} \omega^{2}+O\left(\omega^{4}\right)
$$

we get

$$
\begin{aligned}
W_{\omega}(z) & =-\frac{\ln \omega}{\pi}+\left(\frac{\ln (2 / \pi)}{\pi}+V_{0}(z)\right) \\
& +\omega^{2}\left(\frac{\pi}{24}-V_{2}(z)\right)+O\left(\omega^{4}\right),
\end{aligned}
$$


one deduces the asymptotic relation

$$
\mathcal{T}\left(x_{0}\right)=-\frac{|\Omega|}{\pi D_{h}} \ln \omega+\left(\frac{|\Omega| \ln (2 / \pi)}{\pi D_{h}}+\frac{1}{2 \pi} \int_{\Omega} \frac{d x}{D(x)} \ln \left(\frac{\left|1-\phi_{x_{0}}^{-1}(x)\right|^{2}}{\left|\phi_{x_{0}}^{-1}(x)\right|}\right)\right)+\omega^{2}\left(\frac{\pi|\Omega|}{24 D_{h}}-\int_{\Omega} d x \frac{V_{2}\left(\phi_{x_{0}}^{-1}(x)\right)}{D(x)}\right)+O\left(\omega^{4}\right)
$$

where

$$
\frac{1}{D_{h}}=\frac{1}{|\Omega|} \int_{\Omega} \frac{d x}{D(x)}
$$

We emphasize that the smallness of the harmonic measure $\omega=\omega_{x_{0}}(\Gamma)$ is not related to the smallness of the escape region $\Gamma$. In fact, the harmonic measure characterizes the "accessibility" of $\Gamma$ by Brownian motion starting from $x_{0}$. The escape region can be very small but if $x_{0}$ lies close to $\Gamma$, the harmonic measure is close to 1 (e.g., $\omega=1$ when $\left.x_{0} \in \Gamma\right)$. On the opposite, the escape region can be large but almost "inaccessible" from $x_{0}$ due to diffusion screening, in which case the harmonic measure is small (e.g., $\omega=0$ when $x_{0} \in \partial \Omega \backslash \Gamma$ ).

\section{SM5. GENERATION OF IRREGULAR DOMAINS}

As discussed in SM2 the unit disk can be mapped onto a given polygonal domain by various numerical tools such as a Schwarz-Christoffel transformation [58] or "zipper" algorithm [60]. It is also possible to create irregular domains by means of iterative conformal maps. For an illustrative purpose, we choose this last option and adopt the Hastings-Levitov algorithm for Laplacian growth [28, 29]. This algorithm is based on a conformal map that creates a circular "bump" on the unit disk. Repeating this map iteratively with random bump locations and appropriate rescaling of bump sizes, one can grow DLA-like clusters and study the harmonic measure on its surface. Since this algorithm maps the exterior of the unit disk onto the exterior of the cluster, it is not directly applicable for our purposes as we need a map from the unit disk onto the interior of a bounded domain. Inspired by this algorithm, we consider another basic mapping, from the unit disk onto the unit disk without a nearly semi-circular region:

$\varphi_{\lambda, 0}(z)=\frac{\sqrt{1+\lambda^{2}}}{2}\left(1+z-\sqrt{1-z} \sqrt{\frac{1+z^{2}-2 z \frac{1-\lambda^{2}}{1+\lambda^{2}}}{1-z}}\right)$,

where $\lambda$ is close to the radius of the removed region (see Fig. [44). After $n$ iterations, the conformal map reads

$$
\Phi^{(n)}(z)=\varphi_{\lambda_{1}, \theta_{1}}\left(\varphi_{\lambda_{2}, \theta_{2}}\left(\cdots \varphi_{\lambda_{n}, \theta_{n}}(z) \cdots\right)\right),
$$

where the map $\varphi_{\lambda, \theta}(z)$ specifies the location $\theta$ of the removed region:

$$
\varphi_{\lambda, \theta}(z)=e^{i \theta} \varphi_{\lambda, 0}\left(e^{-i \theta} z\right) .
$$

Both locations $\theta_{k}$ and sizes $\lambda_{k}$ can in general be chosen arbitrarily. To reduce size distortions due to conformal maps and keep physical sizes of all removed regions of the same order $\lambda_{0}$, we set $\lambda_{n}=\lambda_{0} /\left|\Phi^{(n-1)^{\prime}}\left(e^{i \theta_{n}}\right)\right|$, where $\Phi^{(n-1)^{\prime}}$ is the derivative of the map at the previous step $n-1$ which is expressed through the explicitly computable derivatives of $\varphi_{\lambda_{k}, \theta_{k}}$. Note that $\Phi^{(n)}(z)$ maps 0 to 0 . Once the conformal map is constructed (i.e., the sets $\lambda_{1}, \ldots, \lambda_{n}$ and $\theta_{1}, \ldots, \theta_{n}$ are chosen or determined), one can apply the Möbius transformation to ensure the mapping from 0 to a given point $x_{0}$. In fact, it is enough to replace the argument $z$ in Eq. (S66) by its Möbius transform $M_{z_{0}}(z)$ given in Eq. (S33), where $z_{0}$ is the pre-image of the point $x_{0}$,

$$
z_{0}=\left[\Phi^{(n)}\right]^{-1}\left(x_{0}\right),
$$

and this inverse conformal map is obtained as

$$
\left[\Phi^{(n)}\right]^{-1}(x)=\varphi_{\lambda_{n}, \theta_{n}}^{-1}\left(\varphi_{\lambda_{n-1}, \theta_{n-1}}^{-1}\left(\cdots \varphi_{\lambda_{1}, \theta_{1}}^{-1}(x) \cdots\right)\right),
$$

where

$$
\varphi_{\lambda, \theta}^{-1}(x)=e^{i \theta} \varphi_{\lambda, 0}^{-1}\left(e^{-i \theta} x\right)
$$

and

$$
\varphi_{\lambda, 0}^{-1}(x)=x \frac{x-\sqrt{1+\lambda^{2}}}{x \sqrt{1+\lambda^{2}}-1} .
$$

Combining these steps, one gets

$$
\begin{aligned}
\phi_{x_{0}}(z) & =\Phi^{(n)}\left(M_{z_{0}}\left(e^{i \alpha} z\right)\right), \\
\phi_{x_{0}}^{-1}(x) & =e^{-i \alpha} M_{z_{0}}^{-1}\left(\left[\Phi^{(n)}\right]^{-1}(x)\right),
\end{aligned}
$$

where the factor $e^{i \alpha}$ depends on the escape region $\Gamma$ and rotates the disk to ensure that the pre-image of $\Gamma$ is $(-\pi \omega, \pi \omega)$, see Eq. (S32). The great advantage of this method is the very fast computation of the conformal map and its inverse due to explicit formulas.

In the example presented in the main text, we set $\lambda_{0}=0.1, n=27$, and each $\theta_{k}$ is chosen randomly as $(2 \pi / 3) \eta+0.2 \chi$, where $\chi$ is the standard normal variable 


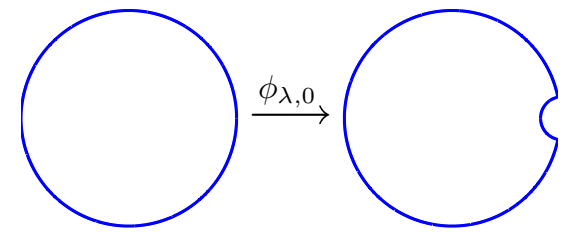

FIG. S4: The function $\varphi_{\lambda, 0}(z)$ maps the unit disk onto the unit disk without a semi-circular region of radius $\lambda=0.2$.

(with zero mean and unit variance), while $\eta$ takes values 0,1 or 2 with equal probabilities. In other words, the algorithm starts from the unit disk and then "digs" three long channels by progressively removing semi-circular regions along three preferred directions $0,2 \pi / 3,4 \pi / 3$.

\section{SM6. SCALING OF THE MFPT WITH THE AREA OF THE DOMAIN}

The MFPT averaged over uniformly distributed starting points is known to be proportional to the area of the confining domain: $\mathcal{T} \propto|\Omega|$ (see [5] and references therein). However, this scaling may not hold when the starting point is fixed. We briefly discussed this issue in the main text by considering an example of a thin long rectangle. Here we extend this discussion and explain when and why the conventional scaling may fail.

We first consider the disk of radius $R$ with an escape arc $(-\epsilon, \epsilon)$ (see SM3A). When the particles start from the origin $\left(x_{0}=0\right)$, Eq. (S40) yields the MFPT

$$
\mathcal{T}(0)=\frac{R^{2}}{D}\left(\frac{1}{4}-\ln \sin (\epsilon / 2)\right),
$$

that indeed scales with the area of the disk. Let now the starting point lie near the boundary, say, at distance $a=R-\left|x_{0}\right|$ such that $a \ll R$. In this case, the Taylor expansion of Eq. (S40) in powers of $a / R$ is

$\mathcal{T}\left((R-a) e^{i \theta_{0}}\right)=\frac{1}{D}\left(\pi R^{2} W_{\epsilon / \pi}\left(e^{i \theta_{0}}\right)+a R U_{\epsilon}\left(e^{i \theta_{0}}\right)+O\left(a^{2}\right)\right)$,

where $W_{\epsilon / \pi}$ is given by Eq. (S25), and

$$
U_{\epsilon}(z)=\frac{1+z}{2 \sqrt{1-2 z \cos \epsilon+z^{2}}} .
$$

If the starting point lies near the escape region (i.e., $\left.\left|\theta_{0}\right| \leq \epsilon\right)$, the function $W_{\epsilon / \pi}\left(e^{i \theta_{0}}\right)$ vanishes, as illustrated in Fig. S1, In this case, the first term in Eq. (S74), which scaled with the area $\pi R^{2}$, disappears, while the next term scales linearly with the radius $R$. In turn, if the starting point is far from the escape region, the MFPT scaling with the area is recovered. We conclude that the scaling with the area is not universal and depends on how far the fixed starting point is from the escape region. Since the fraction of points near the escape region is relatively small, the average of the MFPT over all starting points in $\Omega$ results in the conventional scaling of the global MFPT.

The analysis for arbitrary planar domains is much more involved and goes beyond the scope of this letter. We just mention two possible ways to proceed in this direction.

(i) If the original domain $\Omega$ is dilated by factor 2 and the original starting point $x_{0}$ is similarly transformed into $2 x_{0}$, the conformal map from the unit disk to the dilated domain is twice the original conformal map so that one gets an additional factor 4 from $\left|\phi_{x_{0}}^{\prime}(z)\right|^{2}$ in Eq. (S27) and thus recovers the scaling with the area. However, this argument does not hold if the location of the starting point in chosen differently (e.g., at a fixed distance from the boundary). Using the Möbius transform, one can move the starting point and thus investigate the scaling.

(ii) When the starting point is far from the escape region, the harmonic measure $\omega$ is small, and the logarithmic term in Eq. (S63), which scales with the area $|\Omega|$, provides the dominant contribution to the MFPT. However, if the starting point is close to the escape region, the harmonic measure is close to 1 , and the logarithmic term vanishes. So the main contribution comes from the next term in Eq. (S63) whose scaling needs to be analyzed. In analogy with the disk, a different scaling of the MFPT can be expected in this situation.

\section{SM7. DERIVATION OF THE IDENTITY (S24)}

We consider the series

$$
Q(z, x)=4 \pi \sum_{n=1}^{\infty} \frac{P_{n-1}(x)+P_{n}(x)}{2 n} r^{n} \cos n \theta,
$$

where $z=r e^{i \theta}$. Using the integral representation of Legendre polynomials,

$$
P_{n}(x)=\frac{1}{\pi} \int_{0}^{\pi} d y \nu^{n}(y), \quad \nu(y)=x+i \sqrt{1-x^{2}} \cos y,
$$

we have

$$
\begin{aligned}
Q= & \int_{0}^{\pi} d y\left(1+\frac{1}{\nu(y)}\right) \sum_{n=1}^{\infty}\left(\frac{\left(r e^{i \theta} \nu(y)\right)^{n}}{n}+\frac{\left(r e^{-i \theta} \nu(y)\right)^{n}}{n}\right) \\
=- & \int_{0}^{\pi} d y\left(1+\frac{1}{\nu(y)}\right)(\ln (1-z \nu(y))+\ln (1-\bar{z} \nu(y))) \\
=- & \int_{0}^{\pi} d y\left(1+\frac{1}{x(1-B \cos y)}\right)(\ln ((1-z x)(1-\bar{z} x)) \\
& \left.+\ln (1-A \cos (y))+\ln \left(1-A^{\prime} \cos (y)\right)\right),
\end{aligned}
$$

where $B=-i \sqrt{1-x^{2}} / x, A=i z \sqrt{1-x^{2}} /(1-z x)$, and $A^{\prime}=i \bar{z} \sqrt{1-x^{2}} /(1-\bar{z} x)$. To compute the above integral, 
we use the identity

$$
\int_{0}^{\pi} d y \frac{\ln \left(1-2 a \cos y+a^{2}\right)}{1-2 b \cos y+b^{2}}=2 \pi \frac{\ln (1-a b)}{1-b^{2}},
$$

from which another identity follows

$$
\int_{0}^{\pi} d y \frac{\ln (1-A \cos y)}{1-B \cos y}=\pi \frac{1+b^{2}}{1-b^{2}}\left(2 \ln (1-a b)-\ln \left(1+a^{2}\right)\right)
$$

where $A=2 a /\left(1+a^{2}\right)$ and $B=2 b /\left(1+b^{2}\right)$ or, equivalently,

$$
\begin{aligned}
& a=\frac{1-\sqrt{1-A^{2}}}{A}=\frac{1-z x-\sqrt{1-2 x z+z^{2}}}{i z \sqrt{1-x^{2}}}, \\
& a^{\prime}=\frac{1-\sqrt{1-A^{\prime 2}}}{A^{\prime}}=\frac{1-\bar{z} x-\sqrt{1-2 x \bar{z}+\bar{z}^{2}}}{i \bar{z} \sqrt{1-x^{2}}}, \\
& b=\frac{1-\sqrt{1-B^{2}}}{B}=\frac{1-x}{i \sqrt{1-x^{2}}} .
\end{aligned}
$$

Using the relation (S78), we can compute all integrals separately. We have

$$
\begin{aligned}
& -\frac{Q}{\pi}=\ln ((1-z x)(1-\bar{z} x))-\ln \left(1+a^{2}\right)-\ln \left(1+a^{\prime 2}\right) \\
& +\frac{1}{x}\left[\ln ((1-z x)(1-\bar{z} x)) \frac{1+b^{2}}{1-b^{2}}+\frac{1+b^{2}}{1-b^{2}}\left(2 \ln (1-a b)-\ln \left(1+a^{2}\right)+2 \ln \left(1-a^{\prime} b\right)-\ln \left(1+a^{\prime 2}\right)\right)\right] \\
& =2 \ln ((1-z x)(1-\bar{z} x))+2 \ln (1-a b)+2 \ln \left(1-a^{\prime} b\right)-2 \ln \left(1+a^{2}\right)-2 \ln \left(1+a^{\prime 2}\right) \\
& =2 \ln \left(\frac{\left(1+z-\sqrt{1-2 x z+z^{2}}\right)\left(1+\bar{z}-\sqrt{1-2 x \bar{z}+\bar{z}^{2}}\right)}{(1+x)^{2} z \bar{z}} \frac{(1-z x)(1-\bar{z} x)}{\left(1+a^{2}\right)\left(1+a^{\prime 2}\right)}\right) \\
& =2 \ln \left(\frac{z \bar{z}(1-x)^{2}}{4} \frac{\left(1+z-\sqrt{1-2 x z+z^{2}}\right)\left(1+\bar{z}-\sqrt{1-2 x \bar{z}+\bar{z}^{2}}\right)}{\left(1-x z-\sqrt{1-2 x z+z^{2}}\right)\left(1-x \bar{z}-\sqrt{1-2 x \bar{z}+\bar{z}^{2}}\right)}\right) \\
& =2 \ln \left(\frac{1}{4}\left(1-z+\sqrt{1-2 x z+z^{2}}\right)\left(1-\bar{z}+\sqrt{1-2 x \bar{z}+\bar{z}^{2}}\right)\right) \text {, }
\end{aligned}
$$

from which

$$
Q(z, x)=-4 \pi \ln \left(\frac{\left|1-z+\sqrt{1-2 x z+z^{2}}\right|}{2}\right) .
$$

Comparison of this relation to Eq. (S76) implies the identity (S24). 